\title{
Mutations in the STK11 Gene Characterize Minimal Deviation Adenocarcinoma of the Uterine Cervix
}

\author{
Chie Kuragaki, Takayuki Enomoto, Yuko Ueno, Hongbo Sun, Masami Fujita, \\ Ryuichi Nakashima, Yutaka Ueda, Hiroko Wada, Yuji Murata, Toshihiko Toki, \\ Ikuo Konishi, and Shingo Fujii
}

\begin{abstract}
Department of Obstetrics and Gynecology (CK, TE, YU, HS, MF, RN, YU, HW, YM), Osaka University Faculty of Medicine, Osaka, and Department of Obstetrics and Gynecology (TT, IK), Shinshu University School of Medicine, Matsumoto, and Department of Gynecology and Obstetrics (SF), Kyoto University Faculty of Medicine, Kyoto, Japan
\end{abstract}

\begin{abstract}
SUMMARY: Minimal deviation adenocarcinoma (MDA) is a well-differentiated variant of mucinous adenocarcinoma of the uterine cervix and is found relatively infrequently in the general population. However, MDA is strongly associated with Peutz-Jeghers syndrome (PJS), a rare hereditary autosomal disorder characterized by benign hamartomatous polyposis in the gastrointestinal tract and mucocutaneous pigmentation. A serine threonine kinase gene, STK11, has been identified as the tumor suppressor gene responsible for the PJS. In this study we investigated the possible direct role of STK11 in the development of MDA of the uterine cervix. Eleven rare cases of mucinous MDA, not known to be associated with PJS, were screened for the presence of mutations in the STK11 gene by single-strand conformation polymorphism analysis of PCR-amplified DNA fragments. Subsequently our findings were confirmed with cloning and sequencing. As a control, 24 cases of endocervical adenocarcinomas of other histologic subtypes, with no family history of PJS (19 mucinous adenocarcinomas, 4 endometrioid adenocarcinomas, and 1 clear cell adenocarcinoma), 15 cases of squamous cell carcinomas of the uterine cervix, 5 cases of endocervical glands with pyloric gland metaplasia, and 2 deeply situated nabothian cysts were investigated. Somatic mutations of the STK11 gene were confirmed in $6(55 \%)$ of the 11 mucinous MDAs and $1(5 \%)$ of the 19 mucinous adenocarcinomas, but not in the 5 nonmucinous adenocarcinomas, the 15 squamous cell carcinomas, nor the 5 endocervical glands with gastric metaplasia. MDAs with the STK11 mutation had a significantly poorer prognosis than MDAs without the STK11 mutation $(p=0.039)$. A germline mutation of STK11 was detected in one PJS patient with mucinous adenocarcinoma of the uterine cervix. These results suggest that mutations in the STK11 gene may play an important role in the etiology of MDA of the uterine cervix and may distinguish this rare tumor from other common types of adenocarcinoma of the uterine cervix. (Lab Invest 2003, 83:35-45).
\end{abstract}

$M$ inimal deviation adenocarcinoma (MDA) of the uterine cervix, originally termed adenoma malignum, is an extremely well-differentiated variant of cervical adenocarcinoma representing about $1 \%$ to $3 \%$ of adenocarcinomas of the uterine cervix (Kaminski and Norris, 1983). Microscopically, the cells lining the glands lack cytologic features of malignancy, but the glands are architecturally atypical and varying in size, shape, and location (McKelvey and Goodlin, 1963; Silverberg and Hurt, 1975). Preoperative diagnosis of MDA is difficult because the cervix often appears normal without any obvious lesion grossly or colposcopically (McKelvey and Goodlin, 1963; Silverberg and Hurt, 1975). In nearly all instances, the diagnosis cannot be made reliably based merely on biopsy material but instead requires either a deep cone biopsy or hysterectomy specimen because the depth of penetration of the glands is the key histologic

\section{DOI: 10.1097/01.LAB.0000049821.16698.D0}

Received July 31, 2002.

Address reprint requests to: Dr. T. Enomoto, Department of Obstetrics and Gynecology, Osaka University Faculty of Medicine, 2-2, Yamadaoka, Suita, Osaka 565-0871, Japan.E-mail: enomoto@gyne.med.osaka-u.ac.jp feature of MDA. MDA can be confused with the several conditions in which non-neoplastic glands extend beyond 5 to $7 \mathrm{~mm}$ from the surface (Kaminski and Norris, 1983; Young and Clement, 1991). These MDA-mimicking conditions include endocervical tunnel clusters, deeply situated nabothian cysts, mesonephric hyperplasia, and intestinal or pyloric gland metaplasia (Young and Clement, 1991).

Since the histologic diagnosis of MDA of the uterine cervix is sometimes problematic because of its histologic resemblance to these non-neoplastic conditions, efforts have been made to characterize the histochemical properties of MDA (Gilks et al, 1989; Steeper and Wick, 1986; Toki et al, 1997). Toki et al (1997) showed that MDA lacks expression of characteristic mullerian-type markers, such as estrogen receptor $(E R)$, progesterone receptor (PR), and CA125, and that a fraction of MDA tumor cells contain the gastric epithelial markers, gastric mucin or carcinoembryonic antigen (CEA). Steeper and Wick (1986) reported that positive staining for CEA was helpful in supporting the diagnosis of MDA, but others have shown CEA reactivity only focally in MDA (Gilks et al, 1989; Toki et al, 1997). Ishii et al $(1998,1999)$ and Utsugi et al (1999) 
demonstrated by using the monoclonal antibody HIK1083 that gastric mucin was present in 90\% of MDAs and in $30 \%$ to $57 \%$ of mucinous adenocarcinomas but not in normal endocervical glands. Gastric mucins have been demonstrated not only in the cytoplasm of the MDA tumor cells but also in cervical glands showing a metaplastic change to a pyloric gland morphology, which would make distinguishing pyloric gland metaplasia from an early stage of MDA very difficult (Mikami et al, 1999).

Although MDA is a relatively infrequent malignancy of the female genital tract, accounting for only a small percentage of all uterine cervical adenocarcinoma, it is occasionally associated with the equally rare PeutzJeghers syndrome (PJS), with the result that approximately $10 \%$ of the patients with MDA are reported as patients with PJS (Gilks et al, 1989; Young et al, 1982). PJS is a hereditary autosomal disorder characterized by benign hamartomatous polyposis in the gastrointestinal tract and mucocutaneous pigmentation (Jeghers et al, 1949). Patients with PJS are not only at increased risk for breast, lung, pancreatic, and gastrointestinal cancers (Giardiello et al, 1987) but have an association with unusual types of tumors in the female genital tract, such as the ovarian sex cord tumors, and of course MDA of the uterine cervix (Scully 1970; Young and Scully, 1988; Young et al, 1982). In recent years, the genetic locus of susceptibility for PJS was mapped to chromosome 19p13.3 by linkage analysis (Amos et al, 1997; Hemminki et al, 1997; Nakagawa et al, 1998). The tumor suppressor gene responsible for PJS was subsequently found to be a previously identified gene, $L K B 1$, which encodes a 433-amino acid ubiquitously expressed serine threonine kinase-like nuclear phosphoprotein, referred to as STK11 (Hemminki et al, 1998).

Although a gene responsible for MDA has not been identified, Lee et al (1998) demonstrated that about half of MDAs were affected by loss of heterozygosity at chromosomal marker D19S216 at 19p13.3, within 2 $\mathrm{Mb}$ of the STK11 gene. Their results suggest that independent loss of the STK11 gene could also be responsible for the development of MDA, which is not associated with PJS.

This study was designed to reveal the possible direct role of the STK11 gene in the development of MDA and to associate gene alterations with histologic findings. We screened for STK11 gene mutations by PCR-single strand conformation polymorphism (PCRSSCP) analysis and subsequently confirmed such mutations by cloning and sequencing. We also analyzed MDA tissues for mutations in the K-ras and the p53 genes, two major genes that have been very intensively analyzed in gynecologic cancers. In addition, we assayed for ovarian steroid hormone receptors, CEA, CA125, and gastric gland mucous cell mucin expression by immunohistochemical staining.

\section{Results}

\section{PCR-SSCP and Cloning Sequence Analyses}

We used PCR-SSCP analysis to screen the entire coding region of the STK11 gene for the presence of

mutation in 11 cases of sporadic mucinous MDA, 24 cases of non-PJS endocervical adenocarcinomas of other histologic types, 15 cases of squamous cell carcinomas of the uterine cervix, and 7 benign conditions of the uterine endocervix. Examples of the cases that showed mobility shifts by SSCP analysis are shown in (Fig. 1). To guard against contamination and PCR artifacts, we performed PCR amplification and SSCP-based gel electrophoresis from each DNA sample on at least two separate occasions. Bands with mobility shifts that were observed on at least two separate occasions were considered as candidates for mutation. Mutations were subsequently confirmed by subcloning and sequencing (Fig. 2). The PCR products showing a mobility-shift by SSCP analysis were subcloned into pCR2.1 vectors. DNAs were extracted from multiple individual clones, and PCRSSCP analyses were performed to confirm the these clones showed the same mobility shift as the original PCR products. At least five independent clones were sequenced to determine the presence of mutations. Mutations of the STK11 gene were detected in 6 of 11 sporadic mucinous MDAs and in 1 of 19 non-PJS mucinous adenocarcinomas of the uterine cervix (Table 1). In the seven tumors in which PCR-SSCP analysis suggested the presence of mutations, wildtype bands were undetectable or barely detected, indicating a loss of normal STK11 allele in these tumors. Histology of the MDAs with STK11 mutations is shown in Figure 3. However, no mutations in the STK11 gene were detected in the four endometrioid adenocarcinomas, nor in the case of clear cell adenocarcinoma, nor in the 15 squamous cell carcinomas of the uterine cervix. Mutations were not detected in five endocervical glands with pyloric metaplasias or two deeply situated nabothian cysts either. The frequency of STK11 alterations in MDA is statistically higher than

(A)

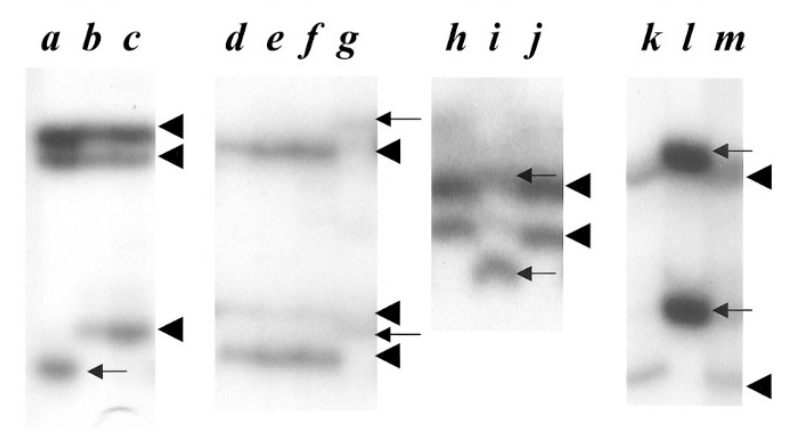

(B)

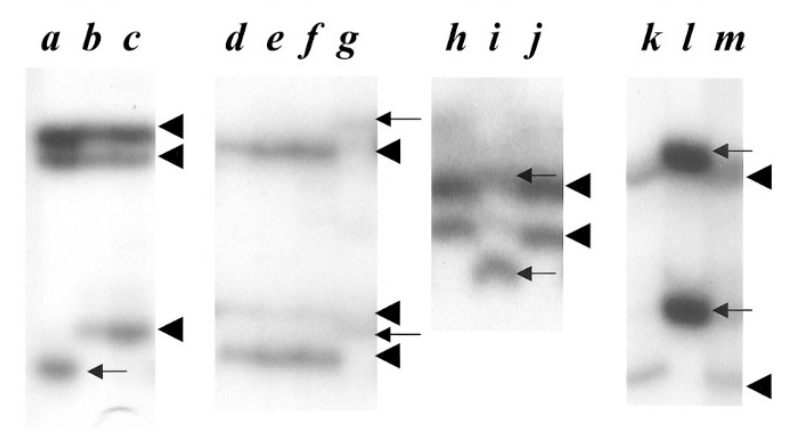

(C)

(D)

Figure 1.

Detection of STK11 gene mutations by SSCP analysis. Each fragment surrounding exon 1 to exon 9 of the STK11 gene was PCR amplified, with incorporation of $\left[{ }^{32} \mathrm{P}\right] \mathrm{dCTP}$. The PCR product was heat denatured and electrophoresed in an $8 \%$ nondenaturing polyacrylamide gel. A, exon 1 ; B, exon 4; C, exon 5; and D, exon 8. PCR products with wild-type STK11 sequences yielded two bands (arrowheads). Bands with mobility sifts (arrow), which suggest the presence of a mutation, were observed in lane a (exon 1 , case 7), lane g (exon 4, case 1), lane i (exon 5, case 3), and lane I (intron 7, case 9), whereas wild-type bands were undetectable or barely detected, indicating the loss of normal STK11 allele in these tumors. 
a

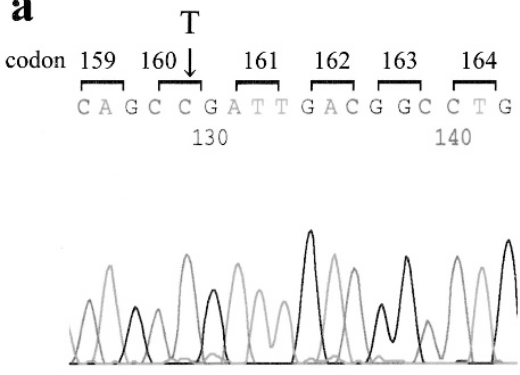

codon 160 b

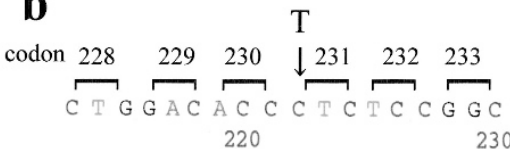

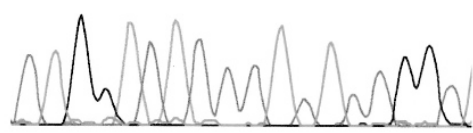

codon 231 c $\overbrace{\text { G T G A A G G A G A T G C T G G A C }}$

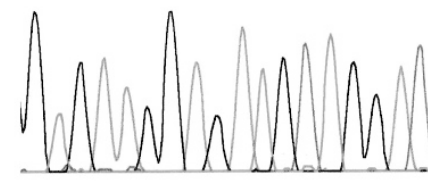

codon 66

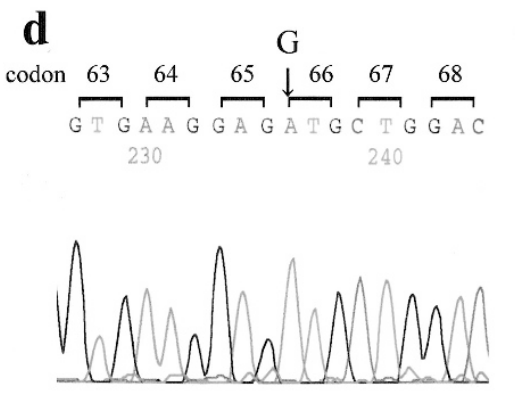

codon 66
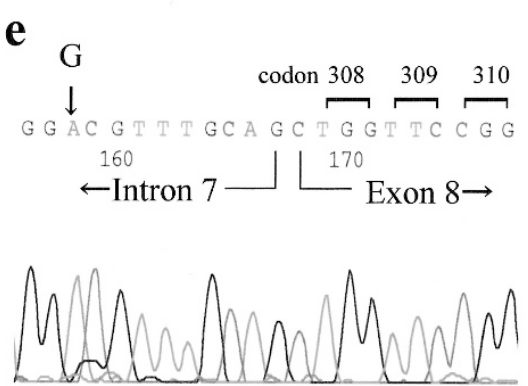

f $\mathrm{G}$

$\underbrace{\downarrow}_{\text {G A A G G GC }} \overbrace{\text { GA G C T G A T G T C G }}^{14}$

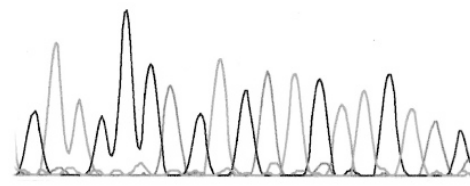

codon 14

Figure 2.

Identification of STK11 somatic mutations in six patients with sporadic minimal deviation adenocarcinomas (MDAs). Genomic sequences are in the $5^{\prime}$ to 3 ' direction, and arrows indicate the location of each mutation. a, CTG [Leu] $\rightarrow$ CCG [Pro] transition at codon 160 in exon 4 in case 1. b, TTC [Phe] $\rightarrow$ CTC [Leu] transition at codon 231 in exon 5 in case 3. $\mathrm{c}$ and d, An identical GTG [Val] $\rightarrow$ ATG [Met] transition at codon 66 in exon 1 in both Cases 7 and 8 . e, G to A alteration in intron 7 located 10 bp upstream of exon 8 in Case 9. f, GAG [Glu] $\rightarrow$ AAG [Lys] transition at codon 14 in exon 1 in Case 11. All mutations were confirmed by sequence analysis of the antisense strand.

Table 1. Frequency of STK11 Mutations in Cervical Lesions

\begin{tabular}{llr}
\hline \multicolumn{1}{c}{ Histologic diagnosis } & \multicolumn{2}{c}{$\begin{array}{c}\text { Mutation } \\
\text { frequency }\end{array}$} \\
\hline Sporadic & & \\
Mucinous MDA & $6 / 11^{a}$ & $(55 \%)$ \\
High grade & $3 / 4$ & $(75 \%)$ \\
Low grade & $3 / 7$ & $(43 \%)$ \\
Mucinous adenocarcinoma & $1 / 19$ & $(5 \%)$ \\
Endometrioid adenocarcinoma & $0 / 4$ & $(0 \%)$ \\
Clear cell adenocarcinoma & $0 / 1$ & $(0 \%)$ \\
Squamous cell carcinoma & $0 / 15$ & $(0 \%)$ \\
Pyloric gland metaplasia ${ }^{b}$ & $0 / 5$ & $(0 \%)$ \\
Deeply situated nabothian cyst & $0 / 2$ & $(0 \%)$ \\
PJS associated & & \\
Mucinous MDA & $0 / 2$ & $(0 \%)$ \\
Mucinous adenocarcinoma & $1 / 1$ & $(100 \%)$ \\
\hline
\end{tabular}

${ }^{a}$ Significantly more frequent than mucinous adenocarcinoma or all other adenocarcinomas except MDA ( $p=0.0045$ and $p=0.0017$ by Fisher's exact test, respectively).

${ }^{b}$ Includes two cases with endocervical hyperplasia and three cases without hyperplasia.

that in mucinous adenocarcinoma $(p=0.0045)$ and in all the adenocarcinomas combined $(p=0.0017)$.

To see whether these mutations were somatic or present in the germ line, the matched normal tissue was studied in cases that contained mutations in the tumor tissue. In sporadic MDAs, no mutations were observed in the matched normal tissue, suggesting that the mutation was somatic. In one of the three tumors that were associated with PJS, analysis of matched normal tissue suggested that the mutation was present in the germ line. The remaining mucinous MDAs associated with PJS did not contain any mutations in the STK11 gene.

In sporadic mucinous MDAs, seven STK11 mutations were detected in six tumors (one tumor, Case 11, contained both a missense mutation and a silent mutation) (Table 2). All seven mutations were singlebase substitutions: four were $\mathrm{G}: \mathrm{C} \rightarrow \mathrm{A}: \mathrm{T}$ transitions, two were $A: T \rightarrow G: C$ transitions, and one was a $\mathrm{G}: \mathrm{C} \rightarrow \mathrm{C}: \mathrm{G}$ transversion. Four mutations were found in exon 1 (Cases 7, 8, and 11), and two of them were an identical GTG $\rightarrow$ ATG mutation in codon 66 (Cases 7 and 8$)$. The remaining three mutations were scattered: one in exon 4 (Case 1), one in exon 5 (Case 3), and the other in the intron 7-exon 8 boundary (Case 3). Of seven STK11 mutations found in sporadic mucinous MDAs, five were missense mutations resulting in an amino acid change, one was a silent mutation in codon 32 (ACC $\rightarrow A C G$ ) resulting in no amino acid change, and the other was a $\mathrm{G} \rightarrow \mathrm{A}$ mutation at $10 \mathrm{bp}$ upstream of the intron 7-exon 8 boundary, which may result in abnormal splicing. A single case of mucinous 

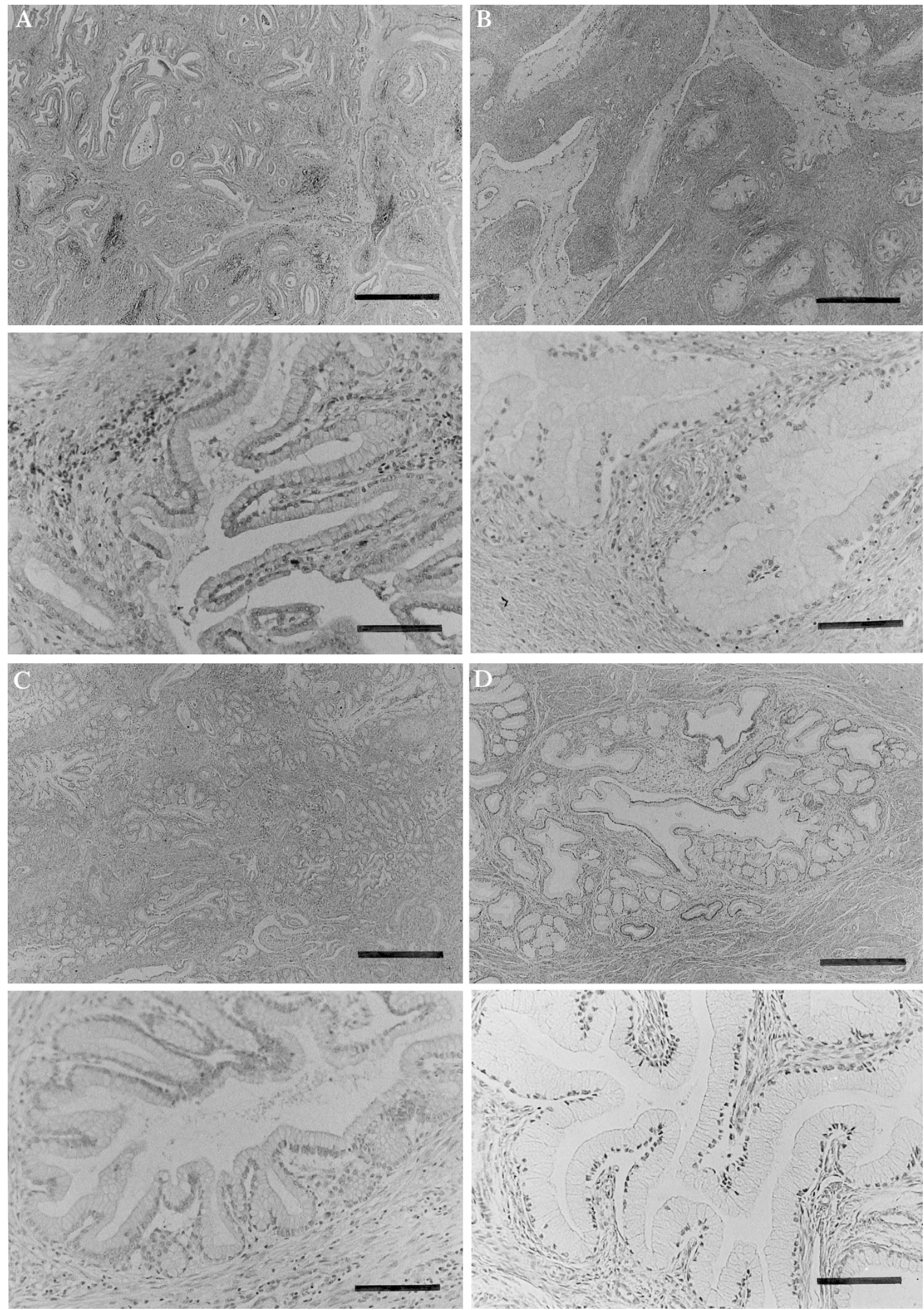

Figure 3.

HE sections of the cases with a STK11 gene mutation are shown. a, Case 1; b, Case 3; c, Case 7; d, Case 8; e, Case 9; and f, Case 11. Both low-power view (top panels) and high-power view (bottom panels) are shown in each case. In each case, cells lining the glands show little atypia; however, the glands are architecturally atypical and vary markedly in size and location, compatible with the diagnosis of MDA. Top panels: Original magnification, $\times 40$; scale bar, $0.5 \mathrm{~mm}$. Bottom panels: original magnification, $\times 200$; scale bar, $0.1 \mathrm{~mm}$. 

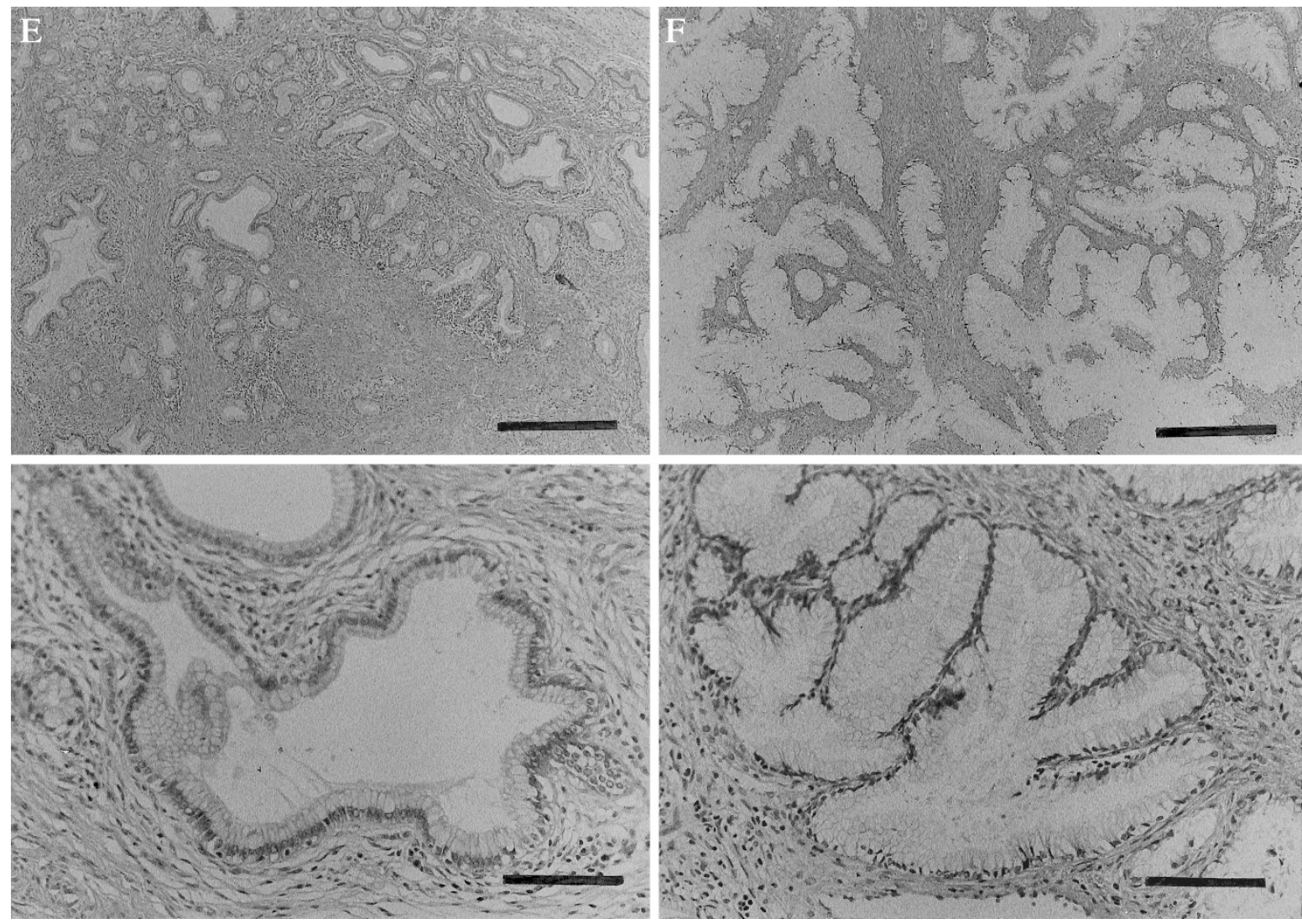

Figure $3 e, f$.

adenocarcinoma of the uterine cervix contained an $A \rightarrow G$ mutation at 2 bp upstream of the intron 3-exon 4 boundary, which may also result in abnormal splicing. In three tumors associated with PJS, one mucinous adenocarcinoma contained an $\mathrm{A} \rightarrow \mathrm{G}$ mutation 2 bp upstream of the intron 7-exon 8 boundary of the STK11 gene, which, again, may result in abnormal splicing.

In 11 sporadic MDAs, we evaluated the association of STK11 mutation with histologic appearance. We found mutation in the STK11 gene in three $(43 \%)$ of seven sporadic MDAs of low grade and three $(75 \%)$ of four sporadic MDAs of high grade.

We also evaluated the association of STK11 mutation with clinical outcome in 11 sporadic cases of MDAs. Four of six patients with MDAs positive for STK11 mutation died within 24 months of the initial surgery, and one of those six had a recurrent tumor, whereas all five of the patients with MDA negative for STK11 mutation were alive more than 50 months after initial surgery. MDAs with STK11 mutation thus had a poorer prognosis than those without mutation (Fig. 4, $p=0.039$, by log-rank test). The six patients with STK11 mutation were diagnosed to be in clinical stage $1 b$, except for one case in stage $2 b$; four of the five cases without STK11 mutation were also diagnosed in stage $1 b$, with one in stage $2 b$. Therefore, there was no significant difference of discernable clinical stage among those with or without STK11 mutation.
A mutation in $p 53$ was detected in only a single case, that of a mucinous adenocarcinoma. A GGC $\rightarrow$ GAC transition was found in codon 245, a reported hot spot for mutation (Cornelis et al, 1997; Würl et al, 1996) resulting in a Gly $\rightarrow$ Asp change. No mutation in exon 1 of $\mathrm{K}$-ras was found in any of the samples.

\section{Immunohistochemical Staining}

Gastric mucin was detected in $10(92 \%)$ of the 11 sporadic MDAs and in both the mucinous MDAs associated with PJS (100\%). Cytoplasmic staining was evenly positive from the surface to deep within the glands in seven cases. In six cases, however, the immunohistochemical reactivity varied for each malignant cell even when they existed in the same neoplastic gland. The staining intensity also differed gland by gland. In the 1 case of MDA that did not express gastric mucin, there was no significant morphologic difference in the tumor glands compared with the other 12 cases that did. In three mucinous adenocarcinomas that were referred to the University Hospital for differential diagnosis of MDA, lesions with tumor cells that resembled MDA were positive for mucin staining but glands with more distinct atypia compatible with typical mucinous adenocarcinomas were negative for staining. All five $(100 \%)$ cases of pyloric gland metaplasia showed strong positive staining of 


\begin{tabular}{|c|c|c|c|c|c|}
\hline \multirow[b]{2}{*}{ Case no. } & \multirow[b]{2}{*}{ Histology } & \multicolumn{4}{|c|}{ Mutation } \\
\hline & & Location & Codon & Sequence & Expected outcome \\
\hline \multicolumn{6}{|l|}{ Sporadic } \\
\hline 1 & Mucinous MDA & Exon 4 & 160 & $\mathrm{CTG} \rightarrow \underline{\mathrm{CCG}}$ & Leu $\rightarrow$ Pro \\
\hline 3 & Mucinous MDA & Exon 5 & 231 & $\underline{\text { TTC }} \rightarrow \underline{\text { CTTC }}$ & Phe $\rightarrow$ Leu \\
\hline 7 & Mucinous MDA & Exon 1 & 66 & $\underline{\mathrm{G}} \mathrm{TG} \rightarrow \underline{\mathrm{A}} \mathrm{TG}$ & Val $\rightarrow$ Met \\
\hline 8 & Mucinous MDA & Exon 1 & 66 & $\underline{\mathrm{GTG}} \rightarrow \underline{\mathrm{A} T \mathrm{~T}}$ & Val $\rightarrow$ Met \\
\hline 9 & Mucinous MDA & 10 bp upstream of intron 7 -exon 8 boundary & & $G \rightarrow \bar{A}$ & Abnormal splicing \\
\hline 11 & Mucinous MDA & Exon 1 & 14 & $\underline{\mathrm{GAG}} \rightarrow \underline{\mathrm{A} A \mathrm{G}}$ & Glu $\rightarrow$ Lys \\
\hline & & & 32 & $\bar{A} C \underline{C} \rightarrow \overline{A C G}$ & Thr $\rightarrow$ Thr \\
\hline 40 & Mucinous adenoca. & 2 bp upstream of intron 3-exon 4 boundary & & $\bar{A} \rightarrow G$ & Abnormal splicing \\
\hline \multicolumn{6}{|c|}{ PJS associated } \\
\hline 29 & Mucinous adenoca. & 2 bp upstream of intron 7-exon 8 boundary & & $A \rightarrow G$ & Abnormal splicing \\
\hline
\end{tabular}

Mucinous adenoca., mucinous adenocarcinoma of the uterine cervix; sporadic, patient without phenotype of PJS in the respective family; PJS associated, patient affected with PJS.

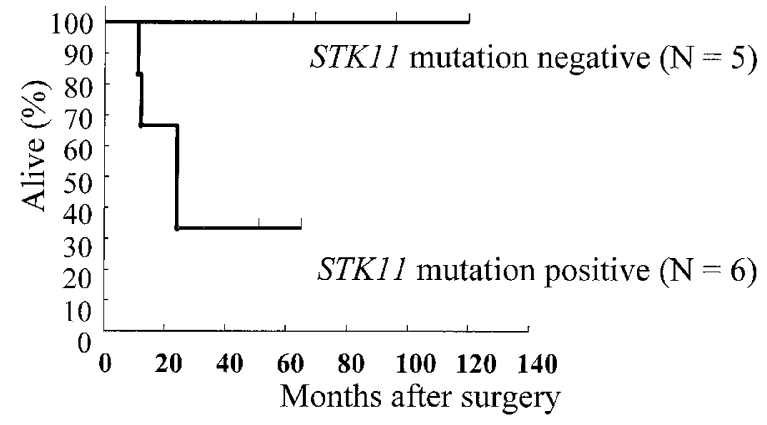

Figure 4.

Overall survival of mucinous MDA patients with and without STK11 mutation. Six patients with STK11 mutation-positive tumors and five patients with STK11 mutation-negative tumors were plotted using the Kaplan-Meier method. Patients with STK11 mutation-positive tumors had a poorer prognosis ( $p=$ 0.039 , by log-rank test).

metaplastic glands. In contrast, two cases of deeply situated nabothian cysts showed negative staining, except for weakly positive cells that were present focally.

Expression of CEA was examined in seven mucinous MDAs, including one PJS-associated MDA, two mucinous adenocarcinomas, and two pyloric gland metaplasias. Positive CEA was detected diffusely or focally in four $(67 \%)$ of six sporadic MDAs, one MDA associated with PJS, one of two mucinous adenocarcinomas, and one of two pyloric gland metaplasias.

We examined the expression of ER, PR, and CA125 in 10 mucinous MDAs, including 2 PJS-associated MDAs, 3 non-PJS mucinous adenocarcinomas, 2 pyloric gland metaplasias, and 2 deeply situated nabothian cysts. ER expression was detected in none of the 10 mucinous MDAs. Expression of PR or CA125 was not apparent in 5 of the 10 mucinous MDAs. The remaining five MDAs expressed either PR or CA125, but only weakly or focally. None of three non-PJS mucinous adenocarcinomas expressed ER, PR, or CA125. In two pyloric gland metaplasias, expression of ER, PR or CA125 was not shown or was apparent only weakly or focally, whereas strong positive ER,
PR, and CA125 expression was shown in the normal cervical glands adjacent to the metaplastic glands of the same histologic section. We evaluated the association of expression of mullerian-type markers (ER, PR, or CA125) with STK11 mutation in 10 MDAs. Three of the four MDAs with a STK11 mutation showed no expression of any mullerian-type markers. Two of six MDAs without STK11 mutation showed no expression of any mullerian-type markers. There was a trend, although statistically significant because of the small sample size, that MDA with STK11 mutation was more likely to lose expression of mullerian-type markers.

\section{Discussion}

Since STK11 was identified as the gene responsible for the PJS, several studies have reported the frequencies and patterns of STK11 mutation in PJS patients compared with similar tumors from patients lacking PJS features (Avizienyte et al, 1998; Bignell et al, 1998; Nakagawa et al, 1999; Resta et al, 1998). However, mutations in the STK11 gene were rarely found in the types of gastrointestinal or breast tumors common to both the general population and patients with PJS (Avizienyte et al, 1998; Bignell et al, 1998; Nakagawa et al, 1999; Resta et al, 1998). In addition, mutations in the STK11 gene were hardly ever found in testicular or ovarian tumors in the general population (Avizienyte et al, 1998; Nishioka et al, 1999).

In this study, we tested for STK11 mutations in a variety of sporadic cervical carcinomas. We detected STK11 mutations in 6 (55\%) of 11 mucinous MDAs and $1(5 \%)$ of 19 mucinous adenocarcinomas but not in 4 endometrioid adenocarcinomas or 15 squamous cell carcinomas. In contrast, we did not detect mutations in K-ras or p53 in mucinous MDAs. Mutations in $\mathrm{K}$-ras or p53 are also reported to be rare in common endocervical adenocarcinoma (Tenti et al, 1998). These findings indicate that STK11 mutation is involved specifically in the development of mucinous MDA. The observation that only about one half of mucinous MDA contained a point mutation in the 
STK11 gene suggests that there may be other mechanisms for STK11 inactivation, such as loss of MDA expression or posttranscriptional regulation of STK11 protein stability in the tumors that lack a STK11 point mutation. Alternatively there may be other genes associated with the mucinous MDA lacking STK11 mutation, because STK11 mutation is detected in only roughly half of patients, even among those with familial PJS (Nakagawa et al, 1998; Resta et al, 1998).

Our observation of a linkage between MDA and STK11 mutation is somewhat in contrast with the previous report by Connolly et al (2000) in which they found no mutations in eight sporadic MDAs. This discrepancy is possibly a result of the differences in methodologies used to detect mutations. They analyzed for the STK11 mutation by direct sequencing of PCR-amplified fragments. We screened for mutations by PCR-SSCP analysis and subsequently confirmed each mutation by cloning and sequencing. PCR-SSCP analysis is much more sensitive for detecting mutated alleles in mixed-cell tumors than is direct sequencing (Enomoto et al, 1995). It is unlikely that the mutations we detected were the result of PCR artifacts because we performed PCR amplification and SSCP-based gel electrophoresis from each DNA sample on at least two separate occasions, and only the bands with mobility shifts observed on at least two separate occasions were further processed for subcloning and sequencing. Moreover, we sequenced at least five clones to determine the presence of mutation. By using the same methodology for all the samples we analyzed, and by repeating the whole experiments at least twice, we confirmed the findings of frequent mutations specifically in mucinous MDA but not in the tumors of other histologic types.

Lee et al (1998) observed allelic loss at chromosomal region 19p13.3 in nine cases of sporadic MDAs. They found allelic loss in all six informative cases at chromosomal marker D19S216, which is located over $3 \mathrm{Mb}$ centromeric to STK11, and in two of three informative cases at chromosomal marker D19S886, which resides $190 \mathrm{~Kb}$ distal to STK11 gene. From these results they suggested that there might be a tumor suppressor gene distinct from STK11 that is involved in the development of sporadic MDA. However the number of samples they analyzed for loss of heterozygosity was too small to convincingly conclude that STK11 was not the principal 19p gene responsible for MDA or that an alternative gene exists. We were unable to perform systematic analysis of loss of heterozygosity using microsatellite markers because of insufficient DNA quantity. However, our SSCP analysis showed that all six MDAs that contained STK11 point mutations also had lost their remaining wild-type allele. This suggests that both alleles of STK11 are inactivated in these six tumors, which strongly supports the proposal that STK11 is the primary 19p gene responsible for the etiology of MDA.

Of the eight mutations of STK11 found in sporadic MDAs or mucinous adenocarcinomas, four mutations were in exon 1, suggesting that exon 1 may be a "hot spot" of mutation for sporadic MDAs. Nakagawa et al
(1998) reported germline STK11 mutations in exon 6 in five of ten PJS patients, and Connolly et al (2000) reported STK 11 mutations within exons 4 and 6 in two PJS family patients with sex cord tumors with annular tubules, whereas Hemminki et al (1998) demonstrated STK11 mutations in exon 1 in seven of twelve PJS cases. Therefore exons 1 and 6 might be the "hot spots" of mutation for familial PJS. Of the eight mutations we identified in sporadic MDAs, five were missense mutations, one was a silent mutation, and two were point mutations at intron-exon boundaries that could potentially result in abnormal splicing. STK11 splicing abnormalities in PJS families have been previously noted (Abed et al, 2001). In contrast, most of the mutations reported in PJS have been nonsense mutations or small insertions/deletions (Hemminki et al, 1998; Nakagawa et al, 1998; Resta et al, 1998). Analysis of additional cases will be required to determine the differences of hot spot and types of mutations between sporadic MDAs and those associated with PJS.

It is of note that we did not detect any STK11 mutations in five cases of pyloric gland metaplasias. These five cases were originally referred to the University Hospital for consultation as to whether they were a very early lesion of MDA or an otherwise benign pyloric gland metaplasia. In these cases, cells resembling the pyloric glands of the stomach were prominent, but there was no distinct nuclear atypia or evidence of stromal invasion. However, it is not well known yet how to distinguish very early stages of MDA (ie, MDA in situ) from pyloric gland metaplasia or whether pyloric gland metaplasia is a precursor of MDA (Ishii et al, 1998; Mikami et al, 1999; Tsuda et al, 2000). Immunohistochemical staining with HIK1083, CEA, ER, PR, or CA125 was uninformative, because both mucinous MDA and pyloric gland metaplasia showed decreased or no expression of the characteristic mullerian-type markers and, instead, expressed gastric epithelial substances, as reported previously (Mikami et al, 1999). The observation of frequent STK11 mutation in mucinous MDA but no mutation in these five cases suggest that these lesions may not be an early stage of MDA but rather benign pyloric gland metaplasia. Further studies will be necessary to identify the natural history of MDA.

The prognosis for MDA is unsettled. Although several studies have suggested an extremely poor prognosis (Gilks et al, 1989; McKelvey and Goodlin, 1963), other series have found survival rates for MDA similar to ordinary well-differentiated adenocarcinoma of the cervix at the same stage (Kaminski and Norris, 1983; Silverberg and Hurt, 1975). A majority of reports found that the survival of patients with MDA is an exception to the general rule that a well-differentiated carcinoma has a better prognosis than a poorly differentiated carcinoma. We showed that mucinous MDAs with an STK11 mutation had significantly poorer prognosis than the tumors without mutation despite the fact that there was no significant difference in clinical stage, histologic grade, or size of the tumor between the two categories (MDA with or without STK11 mutation), 
although mutation was found more frequently in highgrade MDAs than low-grade tumors (3/4 verses 3/7). The fact that all five patients with MDA lacking STK11 mutation survived at least 50 months after the initial surgery suggests that some of these patients may actually have had benign or premalignant conditions rather than true MDA, reminding us that histologic distinction between true MDA and look-alike lesions is very difficult. The trend that MDA with STK11 mutation is more likely to lose expression of mullerian-type markers than MDA without STK11 mutation may also suggest that some of these patients may not have true MDA. Analysis of STK11 mutation thus might be useful for diagnosis and prediction of the prognosis of such cases.

In conclusion, we showed that mutations in the STK11 gene distinguish mucinous MDAs from other common adenocarcinomas of the uterine cervix. Mutation analysis of the STK11 gene may facilitate the differential diagnosis of mucinous MDA from not only welldifferentiated mucinous adenocarcinoma but from benign glandular lesions of which pyloric gland metaplasia is present.

\section{Materials and Methods}

\section{Materials}

Twenty-three formalin-fixed paraffin-embedded tissue specimens were obtained from Shinshu University Hospital, Kyoto University Hospital, Osaka University Hospital, and their affiliated hospitals. These included cases that were originally diagnosed as mucinous MDA and cases that were referred to the University Hospital for differential diagnosis of (1) MDA and well-differentiated adenocarcinoma and (2) early stage of MDA with no obvious stromal invasion and benign pyloric gland metaplasia. Hematoxylin-eosin (HE) sections were re-evaluated by three investigators (S.F., I.K., and T.E.). Identification of MDA was made based on (1) cytologically bland glands that varied in size and shape, (2) increased mitotic activity, (3) a hyperplastic appearance of the glands at the surface, and (4) an increased number of glands positioned deeper than the lower level of normal endocervical glands (Kurman et al, 1992).

Nine cases meeting these four criteria were diagnosed as low-grade MDA. They included two cases that had associated PJS and were affected by intestinal polyposis and oral pigmentation. Four cases also met these criteria in most parts of the lesion, except for focal areas in which the glands had mild cytologic atypia and architectural complexity. These cases were diagnosed as sporadic MDA but of a high grade. Therefore, 11 cases were diagnosed as sporadic MDA. Five cases had small lesions compatible with MDA and thus were referred to the University Hospital, but they also had major lesions with atypical glands compatible with well-differentiated adenocarcinoma. These included one case that arose from a PJS family. This patient was affected by intestinal polyposis and oral pigmentation. These five cases were diagnosed as well-differentiated adenocarcinoma.

Another five cases were referred to the University Hospital for consultation as to whether they were early lesions of MDA or merely benign proliferative cervical glands, in particular those cases that included pyloric gland metaplasia. In these cases, proliferating endocervical glands, lined by columnar cells enriched with mucin, were tightly arranged in clusters with a backto-back appearance, resembling pyloric glands. However, there was no distinct nuclear atypia or evidence of stromal invasion. In addition, the proliferative glands were mixed with nabothian cysts and were not located deeper than 7 to $10 \mathrm{~mm}$ from the surface. We diagnosed these cases as glandular hyperplasias with pyloric gland metaplasia (Mikami et al, 1999). However, we could not rule out the possibility that these lesions may actually have been early lesions or precursors of mucinous MDA.

As controls, we analyzed paraffin-embedded tissue specimens of an additional 20 endocervical adenocarcinomas (15 mucinous adenocarcinomas, 4 endometrioid adenocarcinomas, and 1 clear cell carcinoma), 15 squamous cell carcinomas of the uterine cervix, and 2 deeply situated nabothian cysts obtained from the Osaka University Hospital.

The age of the 11 patients with sporadic MDA ranged from 31 to 50 years, with an average age of 44 years. Clinical stages were $1 \mathrm{~b} 1$ to $2 \mathrm{~b}$ : two patients were diagnosed at stage $2 \mathrm{~b}$, the others were at stage $1 \mathrm{~b}$.

The 13 mucinous MDAs (11 sporadic and 2 associated with PJS), 5 mucinous adenocarcinomas (4 sporadic and 1 associated with PJS), 5 pyloric gland metaplasias, and 2 deeply situated nabothian cysts were also analyzed for mutations in the p53 and K-ras genes.

\section{Laser Capture Microdissection (LCM) and DNA Extraction}

Archival formalin-fixed, paraffin-embedded tissues were cut at $6 \mu \mathrm{m}$ thickness at 10 serial levels and then deparaffinized and stained with methyl-green. Subsequently, the neoplastic gland specimens were precisely dissected by an LCM system (LM200; Arcturus Engineering, Santa Clara, California) (Fig. 5). A carbon

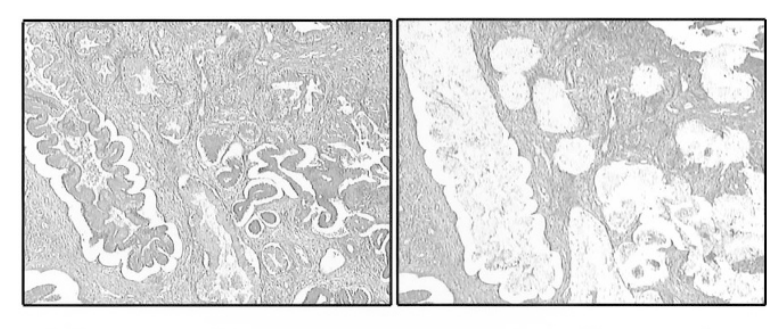

a b

Figure 5

Method of obtaining cervical glands from paraffin-embedded tissue for DNA extraction by a laser capture microdissection (LCM) system. a, Sections derived from Case 7 were stained with methyl green to visualize tumor cells b, Tumor cells were precisely isolated from stromal tissue by LCM system. 
dioxide laser pulse specifically activated a transparent thermoplastic film above the targeted cervical glands and allowed selective dissection of the epithelial cells. Some tumor glands of the specimens adhered too strongly to the slides for transference onto the laseractivated film by the standard method. In such cases, the margins of the tumor glands were etched by surgical blade under microscopy first and subjected to LCM to collect neoplastic cells with the films. DNA was extracted from the microdissected cell specimens by proteinase $\mathrm{K}$ digestion, followed by phenol/chloroform extraction (Mutter and Boynton, 1995). As a matched control, corresponding histologically normal ovarian tissue or normal endometrium of the uterine corpus was used.

\section{PCR-SSCP Analysis}

The 9 exons of STK11 were amplified independently using 13 pairs of primers. The primer sequences and the amplification conditions were as described previously (Bignell et al, 1998; Connolly et al, 2000; Dong et al, 1998; Jenne et al, 1998; Nakagawa et al, 1998). The primer sequences and their annealing temperatures are shown in Table 3. Exon 1 of K-ras and exons 5 to 8 of p53 were also amplified using the primers and the conditions previously published (Enomoto et al, 1991; Fujita et al, 1992). The PCR products had incorporated [32 P]dCTP. After PCR amplification, nine volumes of gel loading buffer (95\% formamide, $20 \mathrm{~mm}$ EDTA, $0.05 \%$ bromphenol blue, and $0.05 \%$ xylene cyanol) were added and the samples were heat denatured at $98^{\circ} \mathrm{C}$ for 5 minutes, chilled on ice, and subjected to electrophoresis on an $8 \%$ nondenaturing polyacrylamide gel at room temperature or $4^{\circ} \mathrm{C}$. The gel was vacuum dried and exposed to Kodak X-Omat film at $-70^{\circ} \mathrm{C} 48$ hours.

We performed PCR amplification and SSCP-based gel electrophoresis from each DNA sample on at least two separate occasions. Bands that underwent SSCP mobility shifts on at least two separate occasions were considered as candidates for having mutations.

\section{Subcloning and Sequencing}

The PCR products showing a mobility shift by SSCP analysis on at least two separate occasions were subcloned into pCR2.1 vectors using the TA Cloning Kit (Invitrogen, Carlsbad, California). DNAs were extracted from multiple individual clones, and PCRSSCP analysis were performed to confirm whether these clones showed the same mobility shift as the original PCR products. At least five clones that showed the same mobility shift were sequenced using a BigDye Terminator Cycle Sequencing Ready Reaction Kit (Perkin-Elmer Cetus, Norwalk, Connecticut) and run on an automatic 377 Genetic Analyzer DNA sequencer (Perkin-Elmer Cetus). Subcloning and sequencing were repeated in duplicate experiments.

\section{Immunohistochemical Analysis}

Expression of gastric mucin, ER, PR, CEA, and CA125 were detected by immunohistochemistry, as previously described (Toki et al, 1997), in 11 sporadic MDA, 2 mucinous MDA associated with PJS, 3 non-PJS mucinous adenocarcinomas, 5 pyloric gland metaplasia, and 2 deeply situated nabothian cysts. Formalinfixed, paraffin-embedded tissues were sectioned at 3 $\mu \mathrm{m}$ and deparaffinized. The sections were hydrated through an ethanol series. Antigen enhancement was performed by microwave pretreatment for 15 minutes in $0.01 \mathrm{M}$ sodium citrate buffer, $\mathrm{pH}$ 6.0. Immunohistochemical staining was performed on serial paraffin sections with the following primary antibodies: antigastric gland mucous cell mucin (HIK1083; Kanto Reagents, Tokyo, Japan), anti-ER, anti-PR (Immunotech, Marseille, France), anti-CEA (II-7; Dako, Kyoto, Japan), and anti-CA125 (Novocastra, Newcastleupon-Tyne, England). The streptavidin-biotin method (Nichirei, Tokyo, Japan) was used for the subsequent

Table 3. PCR Primer Sequences and Annealing Temperatures for PCR-SSCP Analysis of the STK11 Gene

\begin{tabular}{llllc}
\hline & \multicolumn{2}{c}{ Sequence $\left(5^{\prime}-3^{\prime}\right)$} & & $\begin{array}{c}\text { Annealing } \\
\text { temperature } \\
\text { Exon }\end{array}$ \\
\cline { 2 - 3 }$\left({ }^{\circ} \mathrm{C}\right)$
\end{tabular}

References for sequences of PCR primers: Jenne et al, 1998; Nakagawa et al, 1998; Bignell et al, 1998; Dong et al, 1998; and Connolly et al, 2000. 
immunohistochemical procedure (Ishii et al, 1998, 1999; Toki et al, 1997). Only nuclear staining was judged as positive for ER and PR, whereas cytoplasmic staining was judged as positive for gastric mucin, CA125, and CEA.

\section{Statistical Analysis}

The significance of differences in the frequency with and without mutation in histopathologically different categories of lesions was estimated using the Fisher's exact test. The survival curves of mucinous MDA with or without STK11 mutation were plotted by the Kaplan-Meier method, and differences of overall survival were tested using the log-rank test.

\section{Acknowledgements}

The authors are grateful to the following: Dr. Chika Kosaka for excellent technical assistance in immunohistochemical staining; Dr. Tsujimoto, Dr. Suzuki, Dr. Ito, Dr. Arimoto, Dr. Masuhara, and Dr. Yamaguchi for providing material and information on these cases; Dr. Nakamura and Dr. Ozaki for helpful discussions; Mr. Tanaka, Ms. Yukiko Ushijima, and Ms. Nanatsu Nishimura for helpful technical assistance; Mr. M. Hartfield for revision of the text; and Dr. Gregory Buzard for his thoughtful review of the manuscript.

\section{References}

Abed AA, Günther K, Kraus C, Hohenberger W, and Ballhausen WG (2001). Mutation screening at the RNA level of the STK11/LKB1 gene in Peutz-Jeghers syndrome reveals complex splicing abnormalities and a novel mRNA isoform (STK11 c.597 \&caret; 598insIVS4). Hum Mutat 18:397-410.

Amos Cl, Bali D, Thiel TJ, Anderson JP, Gourley I, Frazier ML, Lynch PM, Luchtefeld MA, Young A, McGarrity TJ, and Seldin MF (1997). Fine mapping of a genetic locus for Peutz-Jeghers syndrome on chromosome 19p. Cancer Res 57:3653-3656.

Avizienyte E, Roth S, Loukola A, Hemminki A, Lothe RA, Stenwig AE, Fosså SD, Salovaara R, and Aaltonen LA (1998). Somatic mutations in $L K B 1$ are rare in sporadic colorectal and testicular tumors. Cancer Res 58:2087-2090.

Bignell GR, Barfoot R, Seal S, Collins N, Warren W, and Stratton MR (1998). Low frequency of somatic mutations in the $L K B 1 /$ Peutz-Jeghers syndrome gene in sporadic breast cancer. Cancer Res 58:1384-1386.

Connolly DC, Katabuchi H, Cliby WA, and Cho KR (2000). Somatic mutations in the STK11/LKB1 gene are uncommon in rare gynecological tumor types associated with PeutzJeghers syndrome. Am J Pathol 156:339-345.

Cornelis RS, van Vliet M, van de Vijver MJ, Vasen HFA, Voute PA, Top B, Khan PM, Devilee P, and Cornelisse CJ (1997). Three germline mutations in the TP53 gene. Hum Mutat 9:157-163.

Dong SM, Kim KM, Kim SY, Shin MS, Na EY, Lee SH, Park WS, Yoo NJ, Jang JJ, Yoon CY, Kim JW, Kim SY, Yang YM, Kim SH, Kim CS, and Lee JY (1998). Frequent somatic mutations in serine/threonine kinase 11/Peutz-Jeghers syndrome gene in left-sided colon cancer. Cancer Res 58:37873790 .
Enomoto T, Fujita M, Inoue M, Nomura T, and Shroyer KR (1995). Alteration of the p53 tumor suppressor gene and activation of c-K-ras-2 protooncogene in endometrial adenocarcinoma from Colorado. Am J Clin Pathol 103:224-230.

Enomoto T, Inoue M, Perantoni AO, Buzard GS, Miki H, Tanizawa O, and Rice JM (1991). K-ras activation in premalignant and malignant epithelial lesions of the human uterus. Cancer Res 51:5308-5314.

Fujita M, Inoue M, Tanizawa O, Iwamoto S, and Enomoto T (1992). Alterations of the $p 53$ gene in human primary cervical carcinoma with and without human papillomavirus infection. Cancer Res 52:5323-5328.

Giardiello FM, Welsh SB, Hamilton SR, Offerhaus GJA, Gittelsohn AM, Booker SV, Krush AJ, Yardley JH, and Luk GD (1987). Increased risk of cancer in the Peutz-Jeghers syndrome. N Engl J Med 316:1511-1514.

Gilks CB, Young RH, Aguirre P, DeLellis RA, and Scully RE (1989). Adenoma malignum (minimal deviation adenocarcinoma) of the uterine cervix: A clinicopathological and immunohistochemical analysis of 26 cases. Am J Surg Pathol 13:717-729.

Hemminki A, Markie D, Tomlinson I, Avizienyte E, Roth S, Loukola A, Bignell G, Warren W, Aminoff M, Höglund P, Järvinen $H$, Kristo $P$, Pelin $K$, Ridanpää $M$, Salovaara R, Toro T, Bodmer W, Olschwang S, Olsen AS, Stratton MR, de la Chapelle A, and Aaltonen LA (1998). A serine/threonine kinase gene defective in Peutz-Jeghers syndrome. Nature 391:184-187.

Hemminki A, Tomlinson I, Markie D, Järvinen $H$, Sistonen $P$, Björkqvist A-M, Knuutila S, Salovaara R, Bodmer W, Shibata D, de la Chapelle A, and Aaltonen LA (1997). Localization of a susceptibility locus for Peutz-Jeghers syndrome to $19 p$ using comparative genomic hybridization and targeted linkage analysis. Nat Genet 15:87-90.

Ishii K, Hosaka N, Toki T, Momose M, Hidaka E, Tsuchiya S, and Katsuyama $T$ (1998). A new view of the so-called adenoma malignum of the uterine cervix. Virchows Arch 432:315-322.

Ishii K, Katsuyama T, Ota H, Watanabe T, Matsuyama I, Tsuchiya S, Shiozawa T, and Toki T (1999). Cytologic and cytochemical features of adenoma malignum of the uterine cervix. Cancer 87:245-253.

Jeghers H, McKusick VA, and Katz KH (1949). Generalized intestinal polyposis and melanin spots of the oral mucosa, lips and digits: A syndrome of diagnostic significance. N Engl J Med 241:993-1005 and 1031-1036.

Jenne DE, Reimann H, Nezu J, Friedel W, Loff S, Jeschke R, Müller O, Back W, and Zimmer M (1998). Peutz-Jeghers syndrome is caused by mutations in a novel serine threonine kinase. Nat Genet 18:38-43.

Kaminski PF and Norris HJ (1983). Minimal deviation carcinoma (adenoma malignum) of the cervix. Int J Gynecol Pathol 2:141-152.

Kurman RJ, Norris HJ, and Wilkinson EJ (1992). Classifications of tumors of the lower female genital tract. In: Rosai $J$ and Sobin LH, editors. Atlas of tumor pathology, 3rd Series, Fascicle 4. Tumors of the cervix, vagina, and vulva. Washington DC: Armed Forces Institute of Pathology, 91-93.

Lee JY, Dong SM, Kim HS, Kim SY, Na EY, Shin MS, Lee SH, Park WS, Kim KM, Lee YS, Jang JJ, and Yoo NJ (1998). A distinct region of chromosome 19p13.3 associated with the 
sporadic form of adenoma malignum of the uterine cervix. Cancer Res 58:1140-1143.

McKelvey JL and Goodlin RR (1963). Adenoma malignum of the cervix: A cancer of deceptively innocent histological pattern. Cancer 16:549-557.

Mikami Y, Hata S, Fujiwara K, Imajo Y, Kohno I, and Manabe $T$ (1999). Florid endocervical glandular hyperplasia with intestinal and pyloric gland metaplasia: Worrisome benign mimic of "adenoma malignum." Gynecol Oncol 74:504-511.

Mutter GL and Boynton KA (1995). PCR bias in amplification of androgen receptor alleles, a trinucleotide repeat marker used in clonality studies. Nucleic Acids Res 23:1411-1418.

Nakagawa H, Koyama K, Miyoshi Y, Ando H, Baba S, Watatani $M$, Yasutomi $M$, Matsuura $N$, Monden $M$, and Nakamura $Y$ (1998). Nine novel germline mutations of STK11 in ten families with Peutz-Jeghers syndrome. Hum Genet 103:168-172.

Nakagawa H, Koyama K, Nakamori S, Kameyama M, Imaoka S, Monden M, and Nakamura Y (1999). Frameshift mutation of the STK11 gene in a sporadic gastrointestinal cancer with microsatellite instability. Jpn J Cancer Res 90:633-637.

Nishioka Y, Kobayashi K, Sagae S, Sugimura M, Ishioka S, Nagata M, Terasawa K, Tokino T, and Kudo R (1999). Mutational analysis of STK11 gene in ovarian carcinomas. Jpn J Cancer Res 90:629-632.

Resta N, Simone C, Mareni C, Montera M, Gentile M, Susca F, Gristina R, Pozzi S, Bertario L, Bufo P, Carlomagno N, Ingrosso M, Rossini FP, Tenconi R, and Guanti G (1998). STK11 mutations in Peutz-Jeghers syndrome and sporadic colon cancer. Cancer Res 58:4799-4801.

Scully RE (1970). Sex cord tumor with annular tubules: A distinctive ovarian tumor of the Peutz-Jeghers syndrome. Cancer 25:1107-1121.

Silverberg SG and Hurt WG (1975). Minimal deviation adenocarcinoma ("adenoma malignum") of the cervix: A reappraisal. Am J Obstet Gynecol 121:971-975.

Steeper TA and Wick MR (1986). Minimal deviation adenocarcinoma of the uterine cervix ("Adenoma malignum"): An immunohistochemical comparison with microglandular endocervical hyperplasia and conventional endocervical adenocarcinoma. Cancer 58:1131-1138.
Tenti P, Pavanello S, Padovan L, Spinillo A, Vesentini A Zappatore R, Migliora P, Zara C, Ranzani GN, and Carnevali $L$ (1998). Analysis and clinical implications of p53 gene mutations and human papillomavirus type 16 and 18 infection in primary adenocarcinoma of the uterine cervix. Am J Pathol 152:1057-1063.

Toki T, Shiozawa T, Hosaka N, Ishii K, Nikaido T, and Fujii S (1997). Minimal deviation adenocarcinoma of the uterine cervix has abnormal expression of sex steroid receptors, CA125, and gastric mucin. Int J Gynecol Pathol 16:111-116.

Tsuda H, Okada S, and Kasamatsu T (2000). Is florid pyloric metaplasia different from adenoma malignum? Gynecol Oncol 77:341-343.

Utsugi K, Hirai Y, Takeshima N, Akiyama F, Sakurai S, and Hasumi K (1999). Utility of the monoclonal antibody HIK1083 in the diagnosis of adenoma malignum of the uterine cervix. Gynecol Oncol 75:345-348.

Würl P, Taubert H, Bache M, Kroll J, Meye A, Berger D, Siermann A, Holzhausen H-J, Hinze R, Schmidt H, and Rath F-W (1996). Frequent occurrence of $p 53$ mutations in rhabdomyosarcoma and leiomyosarcoma, but not in fibrosarcoma and malignant neural tumors. Int J Cancer 69:317-323.

Young RH and Clement PB (1991). Pseudoneoplastic glandular lesions of the uterine cervix. Semin Diagn Patho 8:234-249.

Young RH and Scully RE (1988). Mucinous ovarian tumors associated with mucinous adenocarcinomas of the cervix: A clinicopathological analysis of 16 cases. Int J Gynecol Pathol 7:99-111.

Young RH, Welch WR, Dickersin GR, and Scully RE (1982). Ovarian sex cord tumor with annular tubules: Review of 74 cases including 27 with Peutz-Jeghers syndrome and four with adenoma malignum of the cervix. Cancer 50:13841402 